Cahiers de recherches médiévales

\title{
Cinéma, Histoire
}

Autour du thème « johannique »

Françoise Michaud-Fréjaville

\section{(2) OpenEdition \\ Journals}

Édition électronique

URL : https://journals.openedition.org/crm/740

DOI : $10.4000 / \mathrm{crm} .740$

ISSN : 1955-2424

Éditeur

Honoré Champion

\section{Édition imprimée}

Date de publication : 1 juin 2005

Pagination : 285-300

ISSN : 1272-9752

Référence électronique

Françoise Michaud-Fréjaville, "Cinéma, Histoire », Cahiers de recherches médiévales [En ligne], 12 spécial | 2005, mis en ligne le 28 juin 2008, consulté le 15 décembre 2022. URL : http:// journals.openedition.org/crm/740; DOI : https://doi.org/10.4000/crm.740 


\title{
risM
}

\author{
$-24-$ \\ Cinéma, Histoire : \\ Autour du thème «johannique »
}

Sujet traité depuis la fin du XIX ${ }^{\mathrm{e}}$ siècle, dès l'aube du nouvel $\operatorname{art}^{1}$, Jeanne d'Arc reste d'actualité. Chaque année la presse se fait l'écho d'un ou plusieurs projets en gestation ici ou là, de part et d'autre des océans. De ces espoirs, le Centre Jeanne d'Arc d'Orléans recueille parfois la confirmation par des courriers, fax, coups de téléphone et visites des réalisateurs ou de leurs collaborateurs et en garde la trace pour l'avenir, quand bien même la plupart des tournages ne devraient-ils jamais être réalisés ${ }^{2}$. On a parfois l'impression qu'un projet de film chasse l'autre. Mais en vérité le corpus que l'on peut sérieusement étudier n'est pas si large qu'on pourrait le croire: jusqu'à présent moins d'une douzaine des films effectivement tournés (sur la quarantaine dont l'existence est à peu près attestée ${ }^{3}$ ) présente, à un titre quelconque, un intérêt majeur pour l'historienne médiéviste que je suis. En effet, une bonne partie des œuvres du début du $\mathrm{XX}^{\mathrm{e}}$ siècle ne sont plus que des noms ${ }^{4}$ et même si on ne considère que les œuvres qui sont effectivement consultables aujourd'hui, on ne peut traiter de la même façon les bobines qui veulent raconter tout ou partie de la vie de la véritable Jeanne et celles qui prennent ce personnage comme écho de l'histoire personnelle de l'héroïne (cas de Joan of Paris de Stevenson en 1942 et du Début de Panfilov en 1970). Les documentaires utilisant pour matériau les montages de miniatures d'époque médiévale (Enrico, 1956, et Lacassin, 1962 [mais celui-ci fut-il réalisé ?]), de photographies (Jeanne au vitrail de C. Antoine en $1961^{5}$ ) ne peuvent pas non plus être ici pris en compte.

${ }^{1}$ Il est aujourd'hui difficile de trancher : Méliès disait avoir tourné les douze tableaux de sa Jeanne d'Arc en 1897, mais le film ne fut inscrit à son catalogue qu'en 1900 . De 1898 on peut dater les quelques minutes de la Jeanne d'Arc de Georges Hatot.

${ }^{2}$ Le Centre Jeanne d'Arc d'Orléans est un centre municipal soumis aux règles de la communication des documents au public: il va de soi qu'avant le délai légal (30 ou 50 ans selon les cas), la correspondance ne peut être consultée qu'avec l'autorisation des gens concernés.

${ }^{3}$ Voir, à partir de la liste établie par V. Pinel, "Jeanne d'Arc à la télévision et à l'écran ", Jeanne d'Arc à l'écran, Études cinématographiques, $\mathrm{n}^{\circ}$ 18-19, 1962, p. 131-134, les compléments apportés par R. Blaetz, Strategies of containment, Joan of Arc in film, Ph. D., New York University, New York, 1985; Ead., «Jeanne d'Arc au cinéma», Bulletin de l'Association des Amis du Centre Jeanne d'Arc, n 13, 1989, p. 29-39. La bibliographie générale peut être consultée dans N. Margolis, Joan of Arc in History, Literature and Film, a select bibliography, New York, Garland, 1990. Le dernier point sur la question : K. J. Harty, «Jeanne au cinéma», Fresh verdicts on Joan of Arc, éd. B. Wheeler et C. T. Wood, New York, Garland, 1996, p. 237-264 (en anglais). Il faut y ajouter bien évidemment le film de Jacques Rivette Jeanne la Pucelle (1993), et celui de Luc Besson Jeanne d'Arc (1999), sortis après les listes et les travaux précédents.

${ }^{4}$ Les Jeanne d'Arc de Caserini (1901) et de Cappellani (1908, en prévision de la béatification de 1909) une Giovanna d'Arco de Nino Oxilia (1913),

${ }^{5}$ Documentaire pour la télévision dont nous cherchons obstinément la trace, sans succès jusqu'à ce jour.

Cahiers de Recherches Médiévales (XII - XV S.), 12spé, 2005 
Ne nous trompons pas, l'historienne est aussi une spectatrice et spontanément se laisse aller à son humeur, ses préférences et sa culture. Il y a le charme, roublard plus qu'on pense, de l'ancienneté (Méliès, 1899), la réelle beauté des scènes de bataille (La merveilleuse vie de Jeanne d'Arc de Marco de Gastyne, 1928, ou Jeanne d'Arc de Besson, 1999) et l'étrangeté des situations héritées des traditions ironiques $\mathrm{du} \mathrm{XVIII}{ }^{\mathrm{e}}$ siècle, tout à la fois voltairiennes et germaniques (Das Madchen Johanna de Gustave Uciky, 1935). On se laisse surprendre par la curiosité envers des «monstres sacrés » s'affrontant à un rôle qui leur a plus ou moins convenu, telle la Geraldine Farrar, à total contre-emploi pour nos yeux de la fin du XX $X^{\mathrm{e}}$ siècle, proposée par Cecil B. De Mille (deux versions, une française et une américaine, 1917) et pas vraiment plus convaincante, quoique plus moderne, une Michèle Morgan hagiographique filmée par J. Delannoy en 1954. La prestation de Milla Jovovich (Besson, 1999) risque bien de se voir vite rangée parmi les simples succès de curiosité. On se laisse émouvoir par la jeunesse de Simone Genevois, petit prodige qui tournait depuis l'enfance et avait les dix-neuf ans du rôle (1928), et par le grand talent de Sandrine Bonnaire qui en avait une dizaine de plus (1993). Pour rester honnête, on essaie de ne pas anticiper en contemplant la toute jeune Jean Seberg $(1957)^{6}$, dont les images se statufient aujourd'hui en une tragique et anachronique pré-passion suicidaire ${ }^{7}$ Il est assez difficile, enfin, de regarder de nos jours la Passion de Jeanne d'Arc de Dreyer (1928) et le Procès de Jeanne d'Arc de Bresson (1962) en se masquant le cartel «chefs-d'œuvre signés de grands Maîtres » qui les souligne et force à un respect parfois aveuglant. Autrement dit, pour exercer mon métier d'historienne, et en l'occurrence traiter de l'historiographie johannique par le biais du cinéma, comme nous disons dans notre jargon, il me faut passer au delà du plaisir ou de l'agacement du spectateur et aller au document. Je m'attacherai à deux aspects qui me semblent regrouper les différentes discussions que ces films peuvent provoquer et dont nous avons l'écho dans les réactions qui nous parviennent de la part de nos correspondants et amis du Centre Jeanne d'Arc d'Orléans. D'abord l'apparence des choses: la couleur locale, c'est-à-dire l'exactitude des décors et costumes, du langage $^{8}$, des faits historiques : les considérations sont alors valables mutatis mutandis pour tous les films qui traitent des temps médiévaux, d'un Excalibur tout aussi fabu-

${ }^{6}$ « La difficulté dans le film est que sainte Jeanne doit avoir l'air vraiment jeune [...] Au théâtre une femme moins jeune aurait l'air assez jeune à cause de la distance » aurait dit Otto Preminger, cité dans J.-L. Bourget, L'histoire au Cinéma. Le passé retrouvé, Paris, Gallimard (Découvertes-Gallimard 141), 1992, p. 99.

${ }^{7}$ Le film, inspiré de B. Shaw, est quasiment invisible en France depuis des décennies. Preminger en avait conservé une copie qu'il ne voulait, semble-t-il, pas divulguer. On peut le visionner à la bibliothèque du Congrès de Washington et au British Film Institute, la cinémathèque de Londres; quelques versions privées circulent, il est passé à la télévision. Le privilège de l'âge me vaut d'avoir vu, lycéenne vite émue, cette Sainte Jeanne! Ce film a été porté aux nues par certains commentateurs: «Preminger parvient au résultat que visaient, sans l'atteindre, les Dreyer et les Bresson. Son abstraction est incarnée, l'âme de Jeanne, dans Sainte Jeanne, ne détruit pas son enveloppe charnelle; elle en émane comme le parfum émane de la fleur» (M. Mourlet, «Jeanne d'Arc et le cinéma », Spectacle du monde, $\mathrm{n}^{\circ} 19$, août 1978, p. 80).

${ }^{8}$ La Jeanne d'Arc de J. Rivette a été étudiée de ce point de vue par P. Contamine de façon détaillée et fort pertinente (P. Contamine, «Jeanne d'Arc, une sainte au cinéma », L'Histoire, $\mathrm{n}^{\circ} 174$, février 1994, p. 77-79). 
lateur que la matière de Bretagne romanesque dont il est issu aux divers Robin des Bois qui ont bien du mal à s'ancrer dans le temps fort précis du retour difficile de Richard-Cœur-de-Lion de la troisième croisade. Ensuite, j'aborderai la conception du personnage de Jeanne, et là c'est une analyse bien périlleuse en raison de la grande part de charge affective, subjective, dont chacun pare, je devrais dire écrase, l'héroïne. Cette conception commande étroitement le sens du film en son époque, signification pour laquelle il est nécessaire de distinguer la volonté du réalisateur et les interprétations que le temps seul permet d'échafauder.

Quand apparaissent sur un écran moderne les images sautillantes, rayées et faiblement colorées des tableaux animés de Méliès ou les silhouettes fantomatiques de Geraldine Farrar et ses compagnons, on a l'impression tout d'un coup que la toile se plisse aux angles et devient le drap tendu des salles ambulantes. Le charme des « incunables » agit à l'instant et l'on sourit avec ce qu'on prétend être une indulgence compréhensive pour les grands ancêtres, ces Primitifs qui ne manquaient pas de talent. Pourtant le bric-à-brac des costumes et des décors n'y est pas plus irréaliste que l'exactitude revendiquée de Fleming, de Rivette ou de Besson ou le volontaire dépouillement de Bresson. On utilisa en 1899 le lieu même de Domrémy pour tourner le début de l'aventure, comme De Mille fit d'une cathédrale de Reims en photographie le fond de ses scènes du sacre; en revanche on ne peut pas dire que les Tourelles de l'Orléans minable de 1993 satisfasse aux exigences minimales de la conformité historique, pas plus que les murailles, en elles-mêmes superbes, d'une citadelle slovaque (Besson) ou, en 1927, celles d'Aigues-Mortes si reconnaissables. On se rappelle que Cocteau apprécia la «stylisation» des décors de Dreyer qui étaient pure et onéreuse recréation et méprisa les murailles fort authentiques, mais languedociennes, de Marco de Gastyne.

Les modes sont toujours malmenées : longtemps, seule Michèle Morgan présenta une coupe de cheveux s'approchant de la coiffure "ronde» des années 1420$1440^{9}$, mais on n'avait pas été jusqu'à lui raser nuque et dessus des oreilles, en ce style que le médiéviste voit avec amusement revenu présentement à la mode pour les adolescents de 1997-1998 ${ }^{10}$ ! Ce fut fait avec Besson, l'époque s'y prêtait davantage, cependant rien n'étant parfait, voici que Jeanne est très blonde. Quant aux usages du temps, la coupe de la chevelure déjà courte d'Andrée Falconnetti, sauvagement tailladée pour mettre Jeanne en conformité avec l'allure des condamnés à la prison perpétuelle, obéit moins aux besoins d'une symbolique de l'humiliation qu'à l'abandon d'une masculinisation allant de pair avec le costume ${ }^{11}$. Il est vrai que

${ }^{9}$ Il est amusant de constater la parenté de la coupe de cheveux d'Ingrid Bergman en 1948 et celle de la Jeanne proposée par l'illustrateur Grasset - profil très étrange à l'époque - pour le frontispice de la Joan of Arc de Marc Twain (Harper \& brothers, 1896), coiffure qui fut adoptée ensuite par l'actrice pour la vie quotidienne, ce qui eut été impossible avec la coupe «ronde» médiévale.

${ }^{10}$ Justifiée au $\mathrm{XV}^{\mathrm{e}}$ siècle pour l'aristocratie par la forme des bassinets et la façon d'attacher le camail, cette mode l'est peut-être actuellement par les casques de skateboard et de rollers : quid novi sub sole?

${ }^{11} \mathrm{P}$. Bost, La passion et la mort de Jeanne d'Arc d'après le film de Dreyer, Paris, Arthème Fayard, 1928 (Le cinéma romanesque, 7), p. 74: «le geôlier s'avance vers elle, armé de longs ciseaux ; il doit lui couper les cheveux, pour qu'elle soit désormais à la triste mode des pri- 
Dreyer a voulu éluder le réalisme temporel du XVe siècle et fit alors de Jeanne tous les prisonniers de tous les temps soumis à l'horreur des coutumes carcérales. Jean Seberg tondue dès le début de son épopée montre que Preminger a choisi de faire coexister l'exactitude de l'armure de la guerrière avec l'image de la «tête ronde et rase» de la future martyre.

Il faut ainsi faire la part des choix esthétiques et symboliques ${ }^{12}$ des cinéastes et des moyens dont disposaient les costumiers et autres accessoiristes. En mettant de côté les mises en scène des seuls procès, nous ne pouvons voir aujourd'hui que des œuvres qui se piquaient d'une certaine vérité médiévale par la représentation des vêtements et des décors, des harnachements de chevaux, des styles de bataille et des scènes de $\operatorname{cour}^{13}$. On rappelle avec une précision sceptique les cinq cents figurants de Méliès, avec envie les régiments français prêtés à Marco de Gastyne ${ }^{14}$, l'on doute des 43000 pièces, scrupuleusement authentifiées conformes, disait-on à l'époque, utilisées par Cecil B De Mille. Or cette "vérité » est, de façon naturelle, entachée d'abord par la mode populaire correspondant à la date à laquelle le film fut tourné. Nous devons reclasser aujourd'hui la poitrine avachie sous son pourpoint trop long de G. Farrar dans sa geôle, au cœur du contexte des héroïnes plantureuses des premiers Max Sennet et également regarder du côté des photographies de théâtre la silhouette des jeunes premières d'avant la Grande guerre. Les collections de l'Illustration théâtrale ou les cartes postales permettent de constater la conformité des dames de la cour de Chinon et du peuple de Rouen chez De Mille, Gastyne et Uciky, avec les figurants de toutes les reconstitutions historiques du début du siècle, comme en témoignent les images du Théâtre du peuple de Bussang. Le costumier de Sarah Bernardt faisait porter à sa Jeanne le très haut col bordé de feston des élégantes de la Mode Illustrée, flatteur pour les ovales parfaits des débutantes et miséricordieux pour les fanons des actrices, disons moins jeunes ${ }^{15}$. Les dames de l'entourage

sonniers $[\ldots]$ La tete hardie de la jeune guerrière se transforme sous ces coups malhabiles en une tête ronde et rase de prisonnier». P. Champion, Procès de condamnation de Jeanne d'Arc, Paris, 1920-1921, t. I, p. 372 (et traduction, t. II, p. 288) : «Des habits de femmes lui ayant été présentés, elle les revêtit, ayant déposés sur-le-champ ses habits d'homme; elle voulut et permit en outre qu'on lui rasât et enlevât les cheveux qu'auparavant elle portait taillés en rond».

${ }^{12}$ En 1999, Jeanne-Milla vire du blond paille au brun dans la prison de Rouen. Tout ce qui brillait n'était donc point or !

${ }^{13}$ Il faut sans doute mettre à part le court-métrage de Stephen Rumbelow Saint Joan (1977), qui présenterait Jeanne (Monica Buferd) en "proto-fasciste» avec une moustache hitlérienne (R. Blaetz, "Jeanne d'Arc au cinéma », Bulletin de l'Association des Amis du Centre Jeanne $d^{\prime} A r c, \mathrm{n}^{\circ} 13,1989$, p. 38) : je ne l'ai personnellement pas vu.

${ }^{14}$ «Rien n'a été négligé pour faire de la Merveilleuse vie de Jeanne d'Arc un grand film par l'ampleur de la figuration, par les reconstitutions de batailles dans un cadre moyenâgeux pour lequel, une fois de plus, les vieilles murailles de nos cités séculaires ont prêté un secours qu'aucun décor ne saurait remplacer », La Petite Illustration, $n^{\circ}$ 408, 24 novembre 1928.

${ }^{15}$ Voir dans Régine Pernoud, Jeanne d'Arc, Paris, le Seuil, édition de 1981 (illustrée), p. 113. Sarah Bernard, née en 1844, avait dépassé la soixantaine quand elle joua sur scène la Jeanne de Soumet (1909). On est surpris en revanche par la remarquable beauté et l'allure de la jeune fille figurant Jeanne d'Arc à Orléans en 1909, coiffée comme la Jeanne de Grasset, et qui a un visage très préraphaélite (cf. L'Histoire, «Jeanne d'Arc, une passion française », $\mathrm{n}^{\circ} 210$, mai 1997, p. 49). 
royal, chez Marco de Gastyne, ont un regard charbonneux et des nattes passant sous des hennins au ras des sourcils qui n'ont qu'un rapport bien lointain avec les coiffures des élégantes de Van Eyck mais tout des photos de mariage bourgeois des années 1920. Un front haut était alors inesthétique, un point c'est tout. Fleming non plus n'osa pas épiler le crâne des dames de sa cour ! Besson l'a osé et devant Mia Farrow en Yolande d'Aragon sorcière, les spectateurs français se sont souvenus d'une Catherine de Médicis télévisée qui avait eu grand succès !

Les armures, même hors cinéma, sont un inépuisable sujet de controverse qui n'est pas seulement dû à la volonté de prétendre pour les antiquaires de présenter le véritable harnois de Jeanne, mais aussi à l'incapacité où nous sommes encore de dater l'apparition et l'agencement de telle ou telle pièce. Les guerriers de Fleming et de Preminger sont actuellement démodés par la science dans certains détails, mais les camails en métal y ont un poids à l'œil infiniment plus satisfaisant que le jersey textile mal fichu porté par Sandrine Bonnaire, lequel est un défi au bon sens tout en justifiant involontairement qu'on ait évité la coiffure ronde des soldats qui n'aurait eu aucune justification. Le harnachement de la Jeanne de Besson est davantage du temps de Charles VIII que de celui de Charles d'Orléans, les pointes qui hérissent La Hire plutôt de Jérôme Bosch que des ateliers tourangeaux, mais leur poids donne leur authenticité aux marches parfois alourdies des valeureux combattants.

Du détail, un peu spécieux parfois, à l'ensemble, la réinvention des scènes collectives, qu'elles présentent la cour royale, les discussions d'état-major, les divers tribunaux, le sacre, la prison ou la place du marché de Rouen, peut toujours être mise en question. La vie de cour quotidienne de Charles VII paraît à tous coups, sauf chez Rivette, comme un temps de parades et d'orgies et en opposition avec ce que constatent depuis toujours les historiens, disons sérieux. Le sommet est atteint chez Uciky, il est vrai pour des raisons en partie étrangères à la recherche précise de la couleur locale. Dans ce film, les hommes du roi Charles se vautrent le soir du sacre dans le vin et la débauche à l'instar des capitalistes du cinéma soviétique de l'époque et plus tard des nantis oisifs de l'Italie fellinienne. Que la cour du roi Charles entre 1418 et 1436 ait été étriquée et sévère, voire pauvre, et de plus moralement déprimée, ne correspond pas à «l'idée de cour» qui doit au contraire offrir une vie creuse, brillante, ludique et inconsciente. Ingrid Bergman, Simone Genevoix, Milla Jovovich se présentent à Chinon dans une salle bien éclairée, au milieu d'une cour nombreuse et ricanante, en costume d'apparat, aux hommes en pelisses mais aux femmes fort décolletées (nous sommes en mars), le roi, ou celui qui en tient lieu, est assis en permanence sur un trône plus ou moins fleurdelisé ${ }^{16}$. Certes, auprès de Charles VII les intrigues couraient, les amours adultères ou illicites également, mais de façon plus feutrée qu'ostentatoire et l'on y comptait chichement chaque écu dépensé dans une économie de guerre, sous la pression d'un sentiment parfaitement obsidio$\mathrm{nal}^{17}$.

Le sacre était un moment délicat à représenter en raison de sa dimension religieuse et politique : Jeanne d'Arc a été largement revendiquée comme image de la

${ }^{16}$ S'il y avait une vingtaine d'hommes (le conseil du roi et les gardes) dans la salle où Jeanne pénétra, c'est assurément le maximum!

${ }^{17}$ Cependant, dans le film de Fleming il y a une notation à la fois juste et amusante : quelques temps après le sacre, le roi Charles fait remarquer que maintenant il peut porter des vêtements dignes d'un roi! 
droite monarchique française ${ }^{18}$. Cet épisode risquait également de devenir un ennuyeux tunnel par l'exposition d'un rituel incompréhensible à la quasi-totalité du public. Besson a inventé un épisode tellement ridicule à propos de la sainte ampoule que le médiéviste, à la fois ébahi et accablé, ne perçoit plus rien des scènes d'ensemble ${ }^{19}$. Seul Rivette a tenté d'aller au-delà de la mécanique de gestes sélectionnés, plus loin qu'une messe en grande pompe reconstituée d'après les gravures de l'époque classique, voire du sacre de Napoléon peint par David. Avec courage, il a fait parcourir aux acteurs et aux spectateurs les différentes étapes de la cérémonie largement télescopées par tous les autres réalisateurs qui tentèrent le grand spectacle de la foule criant Noël, Vivat rex! Il est dommage que Rivette n'ait pas fait passer une émotion collective, une vibration de l'air dans sa cathédrale apparemment glacée (on était en juillet...).

Du procès, on sait l'épure qu'en proposa Dreyer, réduisant le décor aux murs blancs, les mois d'interrogatoires à l'ultime journée, la couleur locale aux coules des juges et à quelques casques. L'efficacité en fut à l'époque remarquable. Mais aujourd'hui la galerie des portraits physiques des juges, tous plus hideux et grotesques les uns que les autres, caricatures nécessaires parce que le muet ne pouvait prouver la duplicité, l'indifférence, la cruauté ou l'acharnement que par les mimiques et la compréhension immédiate de l'âme des protagonistes à travers leur physique, ne passe plus si aisément. Nous ne devrions pas sourire de la laideur obèse de Cauchon mais en avoir peur ${ }^{20}$. Les fugitives images de Jean Massieu, le prêtre huissier aux beaux yeux aussi clairs que son âme $e^{21}$, ne sont maintenant sorties du lot, figées à jamais et popularisées, qu'en raison de la longue Passion qu'allait subir à son tour son interprète, Antonin Artaud, au cours des vingt années qui suivirent. Ce qui saute

\footnotetext{
${ }^{18} \mathrm{G}$. Krumeich, Jeanne d'Arc à travers l'histoire, Paris, 1993, chapitre 5 : « Jeanne d'Arc dans la querelle des deux France», p. 177-244 ; N. Margolis, "La chevauchée solitaire du professeur Thalamas: rationalistes et réactionnaires dans l'historiographie johannique (19041945) ", Bulletin de la Société des Amis du Centre Jeanne d'Arc, n 15, 1991, p. 7-28.

${ }^{19}$ Rappelons la version Besson/Birkin : Yolande d'Aragon (qui ne se trouvait pas en réalité au sacre de son gendre) fabrique en sacristie un saint chrême avec de l'huile qu'elle sort d'on ne sait où, en présence - consentante! - de l'archevêque de Reims. On sait que le chrême est béni chaque année le Jeudi saint et que le saint chrême du sacre était une infinitésimale partie du baume gardé à l'abbaye Saint-Remi de Reims, mélangé avec une quantité appréciable du chrême de l'année. En outre, le fait qu'une femme laïque ait pu trafiquer tout cela sur un autel consacré est proprement inimaginable.

${ }^{20}$ L'effet recherché par Dreyer n'est pas d'accuser le solennel ridicule, comme pour le monstrueux cardinal Bertrand interprété par Lucien Bodard dans Le nom de la Rose de JeanJacques Annaud (1986), mais bien de mettre en valeur une méchanceté, fille de la lourdeur de l'esprit et du corps. L'évêque Cauchon était un esprit cultivé et fin qui choisit mal son camp, ce qui est arrivé, avant et après lui, à bien d'autres « intellectuels » à l'intelligence pourtant aiguë.

${ }^{21}$ Le témoignage de Massieu, donné lors de la procédure en annulation (1455-1456), est à manier avec grande précaution en ce qui concerne la définition de son rôle et l'analyse de ses sentiments à l'époque de la mort de Jeanne, comme tous ceux des protagonistes du procès de Rouen de 1431. Il y eut sans doute de sérieux examens de conscience... Massieu en 1431 avait-il vraiment ce « rayonnement d'archange » attribué en 1962 à l'Antonin Artaud de 1927 (J. Sémolué, «La Passion de Jeanne d'Arc, prise de conscience de Carl Th. Dreyer» Études cinématographiques, $\mathrm{n}^{\circ} 18-19,1962$, p. 41).
} 
aux yeux aujourd'hui pour l'historienne, c'est que, plus encore que ne le disaient certains critiques ${ }^{22}$, Dreyer a effectivement réutilisé à sa manière les formules des peintres et des imagiers (c'est-à-dire les sculpteurs) du nord et de l'est de l'Europe $\mathrm{du} \mathrm{XV}^{\mathrm{e}}$ siècle, et donné à ses juges les trognes des tourmenteurs du Christ ${ }^{23}: \mathrm{c}^{\prime}$ est en vérité une forme de respect de la couleur locale vue par le Moyen Âge lui-même ! La vraisemblance n'y trouve en rien son compte. Pour l'effet d'art et l'émotion obtenue, c'est à la sensibilité et à la culture des spectateurs qu'il faut s'en remettre.

En choisissant une disposition au tribunal - aspect dont nous ignorons tout-, Fleming a repris l'organisation spatiale des lits de justice royaux français, en particulier celui de 1458 représenté par Jean Fouquet, où l'accusé n'apparaît d'ailleurs pas $^{24}$. Le cinéaste américain, comme Dreyer, est néanmoins tombé dans la facilité de donner à l'évêque de Beauvais qui préside ${ }^{25}$ l'aspect évoqué par son nom; il le montre le plus souvent en très gros plan ou de profil, afin d'accuser l'enflure d'un personnage évidemment négatif. Il va de soi que nous ne savons pas grand-chose de l'aspect physique de Cauchon. Preminger, de son côté et par opposition au film précédent, s'est offert le plaisir de le rendre ascétique, dur et blanc comme du marbre (il est interprété par Anton Walbrock), vaguement nazi pour tout dire. De son côté, Bresson a également choisi, cela très clairement pour se démarquer de Dreyer, de présenter un accusateur mince et élégant, un monstre froid (J.-Cl. Fourneau).

Les deux cinéastes américains Fleming et Preminger ont presque tout au long du procès, comme les pièces de théâtre dont ils s'inspirèrent, usé, on n'ose dire abusé car Jeanne était bien isolée en effet, du contraste entre un cordon ininterrompu

${ }^{22}$ Voir en particulier le dossier publié par L'Avant-scène, Cinéma, n ${ }^{\circ} 531$, janvier 1970, p. 5154.

${ }^{23} \mathrm{C}^{2}$ 'est ce que l'histoire de l'art actuelle relève pour la fin du $\mathrm{XIV}^{\mathrm{e}}$ siècle dans des œuvres comme les Petites heures du duc de Berry (v. 1380), où les images de la passion du Christ présentent «une rare combinaison d'actions mobiles et dramatiques, de types ou de gestes grotesques ou pathétiques mais aussi de drapés doux et mélodieux » (Jan Bialostocki, L'art du $X V^{e}$ siècle, des Parler à Dürer, Paris, 1993 [éd. française d'un texte de 1989], p. 96). La même idée a été exprimée par A. Châtelet et R. Recht, Le monde gothique, Automne et renouveau, 1380-1500, Paris, 1988 (L'univers des formes), p. 263 et suivantes, à propos du premier réalisme germanique : "[à la différence du réalisme flamand] le réalisme germanique n'est pas celui des détails, ce n'est pas non plus celui de l'espace, [...] c'est celui des types volontiers vulgaires, celui des gestes souvent violents. La flagellation du Christ (de Maitre Francke, v. 1425) est prétexte à une insistance sur les verges, les visages caricaturaux des bourreaux ». Après 1430, la plupart des peintres du Nord, bien avant Bosch, utiliseront les grimaces et les gestes désordonnés de la populace, non sans un évident antisémitisme-correspondant, si j'ose dire, à l'anticléricalisme fréquent chez les partisans de Jeanne d'Arc-, en contrepoint à la présence pathétique du Christ qui conserve une attitude élégante dans la souffrance et l'humiliation. Les luthériens, tradition religieuse dont relevait Dreyer, sont beaucoup moins intransigeants sur les représentations évangéliques que les calvinistes.

${ }^{24}$ Le duc Jean d'Alençon (1402-1476), compagnon de Jeanne d'Arc et cousin du roi, fut condamné à mort pour haute trahison en 1458 et gracié. Fouquet a représenté pour un exemplaire des Cas des nobles hommez et femmes malheureux de Boccace $(\dagger 1375)$ un lit de justice que l'on a identifié, par les portraits des assistants, comme celui du jugement du duc d'Alençon tenu à Vendôme, auquel l'artiste a pu et dû assister (Munich, Bayerische Staatsbibliothek, Cod. Gall. 369, fol. 2v).

${ }^{25}$ En réalité, la seconde partie des séances (du 27 mars au 24 mai 1431) a été présidée et menée par Jean de la Fontaine, systématiquement absent des écrans. 
d'hommes, les uns assis en grandes robes, les autres debout en arme, cernant totalement la petite escabelle sur laquelle vient misérablement s'asseoir Jeanne. La richesse des couleurs arborées par l'assistance chez Fleming accuse encore la simplicité et la solitude de la Pucelle. Les procès ordinaires médiévaux dont nous avons des images nous montrent le juge seul assis sur un siège à dossier, les notaires accroupis ou sur un tabouret bas avec leurs notes sur les genoux (parfois sur une table basse) et tous les autres, assistants et parties, debout ${ }^{26}$. Les séances n'étaient pas les marathons de nos grands spectacles d'assises.

Les dialogues du procès de Rouen sont historiquement les éléments les plus authentiques de l'histoire de Jeanne d'Arc, les choix faits par les auteurs paraissent toujours arbitraires aux historiens qui ne retrouvent jamais la fort subtile progression des sessions vers le but déterminé d'une condamnation, sinon chez Bresson. Le mécanisme des interrogatoires en boucle, revenant sur eux-mêmes pour faire surgir les contradictions, était peu cinématographique et même peu théâtral. Passons sur les films muets, victimes du progrès. G. Ucicky supprime presque totalement le procès dans une élision désinvolte ${ }^{27}$ sans aller aussi loin qu'un de ses modèles, Schiller, qui arrêtait l'aventure de son héroïne devant Reims ${ }^{28}$. Rivette ne s'attarde guère plus, d'abord parce qu'il a privilégié une longue reconstitution de l'interrogatoire de Poitiers sur laquelle il pouvait tout inventer ou presque, et ensuite en raison d'un scénario qui fait intervenir régulièrement les témoins du procès d'annulation. On peut supposer que, pour lui, il y avait trop de monde autour de Jeanne dans le tribunal rouennais et que cela ne correspondait pas à la conception des scénaristes d'une Jeanne renfermée dans sa douleur et sa prière ${ }^{29}$. L'invention du scénario de Luc Besson de donner à Jeanne une conscience qui la juge dans sa prison est audacieuse, psychologiquement très fine mais ce juge inattendu pose à l'héroïne des problèmes précis auxquels celle-ci ne pouvait pas précisément se heurter de la manière dont le cinéaste les présente ${ }^{30}$.

\footnotetext{
${ }^{26}$ On trouve une série de représentations de tribunaux, y compris des Grands Jours dans la disposition du lit de Justice avec les assistants debout derrière des barrières, dans le manuscrit du Vieux coustumier de Poictou (éd. René Filhol, Bourges, 1956, d'après le ms 18 de la B. M. de Niort, de la seconde moitié du XV $\mathrm{XV}^{\mathrm{e}}$ siècle).

${ }^{27}$ H. Steinberg, «Das Mädchen Johanna de Gustav Uciky ou Jeanne et Goebbels », Études cinématographiques, $\mathrm{n}^{\circ} 18-19,1962$, p. 54-56. Résumé du film : Jeanne est brûlée juste avant que son champion - Maillezais, personnage inventé - n'accoure pour la délivrer. Le procès est inutile parce que la mort de Jeanne est décidée aussi par le roi de France Charles VII qui veut faire de son martyre un nouvel aiguillon du sursaut national français contre les Anglais.

${ }^{28}$ Dans La Pucelle d'Orléans de Schiller (1801), Jeanne n'est pas brûlée mais libérée par ses amis d'Isabeau de Bavière qui la retenait prisonnière et elle est blessée à mort au cours du combat victorieux des Français contre les Anglais devant Reims.

${ }^{29} \mathrm{C}$ 'est, semble-t-il, également l'interprétation de C. Nevers dans «L'avenir d'une illusion», Les Cahiers du cinéma, $\mathrm{n}^{\circ}$ 476, février 1994, p. 25 : «[Le procès est] trop exposé, trop public pour un cinéaste que seul le secret obsède»

${ }^{30}$ Le choix de Dustin Hoffman pour incarner cet interlocuteur fantôme, provocateur comme le reste, n'est pas en cause. C'est l'inadéquation entre les épisodes proposés par Besson à l'examen de conscience de Jeanne (par exemple l'épée de Fierbois) et les séquences de sa vie, telles que la tradition nous les a transmises, qui peut empêcher le spectateur d'éprouver de la compassion (au sens propre) pour la Pucelle.
} 
Bresson est certainement le plus fidèle à la lettre et au verbe, à la suite logique des questions, à la pesanteur du temps, au langage des gestes et c'est ce qui lui a valu, paradoxalement, les critiques les plus féroces pour un film qui aurait regardé "par le trou de serrure ${ }^{31}$ et demeure poignant et oppressant par la mécanique implacable qu'il expose.

Reste ce qu'on ne peut montrer, l'expérience mystique, les «voix », les prières et la mort. La silhouette armée de saint Michel dans la brume du jardin de Domremy, telle que Cecil $\mathrm{B}$. De Mille la montre, après Méliès et avant Marco de Gastyne, fait bricolage de patronage et aucunement révélation divine. Le vitrail explosé de Besson et sa Trinité dans les bois portent fortement le souvenir des tableaux de S. Dali, non des meilleurs. Il a été longtemps difficile d'échapper à l'himmlisches Blick, le regard au ciel imposé par l'iconographie des saints depuis la Renaissance et surtout l'âge baroque et que le Moyen Âge ne pratiqua que rarement. Michèle Morgan, les yeux clos sous l'improbable rayon de lumière de l'église de Lagny qui les éclaire, n'échappe pas non plus à la fadeur sulpicienne la plus éculée (Destinées de J. Delannoy, 1954). Sur les représentations médiévales, les donateurs en prière et les saints patrons qui les présentent regardent devant eux, car c'est là que se trouvent les objets de leur dévotion: Marie et l'enfant Jésus, le plus souvent accessibles à leur niveau humain. Rien n'est plus anachronique pour cette époque que de montrer le blanc des yeux, attitude réservée fort logiquement à la scène de l'Ascension et parfois de la Pentecôte. Mais il est vrai que les conversations sacrées, familières, ne peuvent plus être montrées comme le faisaient les peintres du XVe siècle et que nous nous heurtons à une barrière mentale infranchissable. Il existe, par exemple, une célèbre gravure du «Maître E. S. », dite Le grand jardin, qui montre la Vierge assise dans un jardin (disposé d'ailleurs comme un lit de justice). Distraite de son livre, Marie lève les yeux un instant en tournant la page et nous regarde, les saintes de Jeanne d'Arc, Marguerite tenant en laisse son dragon et Catherine, sa roue auprès d'elle et une rose à la main, surveillent sans l'entraver l'enfant Jésus caracolant sur un bâton et dont le chiot familier tire la robe. Des anges derrière le mur jouent du luth et de la harpe. La paix extraordinaire qui émane du quatuor est presque palpable, en dépit de l'irréalisme absolu de la situation; il paraitrait naturel que Jeanne s'accoudât au muret et demandât quelques nouvelles du paradis.

Il est bien évident que filmer une Jeanne conversant avec ses voix de cette façon $^{32}$, avant le fracas de batailles bien réalistes et un procès méthodique semble un mélange des genres presque impossible aujourd'hui, bien que les Aliens et autres Ghost! et Ghosthunters fassent salles combles. Et Besson est finalement revenu aux nuages des Verts pâturages comme aux trucages de Mélies, numérisés. Rossellini a proposé une version faussement naïve de l'iconographie pieuse pour sa Giovanna

\footnotetext{
${ }^{31}$ Tragédie « regardée par un trou de serrure », dit M. Mourlet, op. cit., p. 80, qui ne donne pas l'origine de sa citation. Cette image est reprise par K. J. Harty: «Bresson's technique is almost voyeuristic, the film displays a fascination with doorways and peepholes ", New verdicts..., p. 255.

${ }^{32}$ Après avoir eu très peur la première fois, comme elle le dit aux juges de Rouen, Jeanne semble avoir eu avec ses conseils (comme elle appelle le plus souvent les voix) des relations empreintes de calme et de confiance. Il n'y a aucune manifestation extérieure délirante.
} 
d'Arco al Rogo (1954), l'oratorio Jeanne au bûcher de Claudel et Honegger ${ }^{33}$. Il n'hésita pas à reprendre les rondes d'anges de Fra Angelico, vues en plongée ou de côté dans les brumes, et à jouer de l'irréalisme total avec Frère Dominique et Jeanne regardant les événements depuis une zone intermédiaire entre ciel et terre. Il y a quelques rappels du Magicien d'Oz... La fantaisie poétique et parfois forcée du texte oblige à prendre le recul de l'imagerie. On doit accepter qu'Ingrid Bergman disparaisse, sans trace de brûlure sur sa robe blanche, vers la Grande Ourse et finisse en étoile comme le Petit Prince. L'historien et l'histoire sont mis knock-out, c'est une réussite du genre.

Les solutions raisonnables de J. Rivette, cinéaste qui laisse en paix à l'écart sa Jeanne en prière, ne laissent pas percevoir les sources d'une foi si forte qu'elle la pousse à l'action, qu'elle lui donne la force de quitter sa famille, d'affronter une société inconnue, braver les interdits physique et vestimentaire, de vivre la vie des camps, surtout de choisir finalement sa mort.

Le charisme de Jeanne peut-il être dirigé et interprété? La solution n'a pas encore été trouvée en dépit du talent ou de la franchise des actrices et des efforts des cinéastes. Plus que la vérité historique, c'est le personnage de l'héroïne en son temps et en celui du cinéma qui est en question.

L'historien doit reconnaitre le droit aux cinéastes d'être des créateurs et de présenter à leur idée le personnage de Jeanne tel qu'ils l'imaginent ou pensent qu'un public peut l'aimer. Les tabous sont stérilisants, au cinéma comme ailleurs. En retour, l'examen critique doit être permis dans la mesure ou les réalisateurs présentent leurs travaux à des êtres pensants.

Dans la conception des diverses «Jeanne », les scénaristes portent une lourde responsabilité, c'est pourquoi la Jeanne au bûcher de Claudel-Honegger-Rossellini fut la plus imaginative et la plus intemporelle. L'histoire n'a aucune prétention au réalisme, elle approche la vérité par des séries de saynètes présentées dans des «maisons» qui s'ouvrent l'une après l'autre dans des nuages d'étoupe blanche, rose et bleutée comme dans les mystères médiévaux, elle n'offre pas vraiment de déroulement chronologique explicatif ${ }^{34}$. Par intérêt et égoïsme, les grands ont déçu les espoirs des humbles et ont sacrifié Jeanne, une fille issue du peuple. Dieu qui est toute intelligence et tout amour distingue le vrai du faux, le juste de l'injuste, le simple du frelaté ; la mort de Jeanne est sa victoire et entraîne le salut des justes, du peuple et de la France malgré les grands, laïcs et clercs. L'œuvre originale avait été représentée en mai 1939 à Orléans, certes pour la fête de la $510^{\text {e }}$ commémoration de la délivrance de la ville ${ }^{35}$, mais dans un contexte lourd des menaces de guerre et une

\footnotetext{
${ }^{33}$ Voir Michel Estève, «Jeanne au bûcher de Roberto Rossellini : les séductions de l'oratorio filmé ou le merveilleux contre le surnaturel », Études cinématographiques, n 18-19, 1962, p. 65-71.

${ }^{34}$ Paul Claudel, Jeanne d'Arc au bûcher, Paris, Gallimard-N. R. F., 1939, introduction : « ainsi les mourants, dit-on, voient à la dernière heure se déployer tous les événements de leur vie, à qui sa conclusion imminente confère un sens définitif ».

${ }^{35}$ Orléans a célébré, plus ou moins somptueusement, sa fête de la délivrance depuis 1430, à l'exception de quelques années de la Révolution (1793-1803) et de la Monarchie de Juillet.
} 
infâme polémique sur l'interprétation de Jeanne par une juive, Ida Rubinstein ${ }^{36}$. Rossellini reprit et filma l'oratorio en offrant à Ingrid Bergman une présentation de l'héroïne dans un registre différent de sa première prestation mais où Ingrid n'est cependant, en miroir, que le double physique absolu de la Jeanne de Fleming avec la même coupe de cheveux, la même robe-suaire de gros tissu blanc ${ }^{37}$. L'innocence incomprise était aussi celle du couple Rossellini-Bergman devant les autorités tant hollywoodiennes qu'italiennes et le film une sorte de défi aux puritains américains et aux curés italiens, par Claudel et Honegger interposés. Le film ne crée pas pour autant un nouveau personnage par rapport à l'original scénique.

Les autres films qui présentent Jeanne d'Arc se répartissent pour l'historien en plusieurs groupes. On peut distingue d'abord les vies complètes subdivisées en deux époques : avant et après Bernard Shaw. Ensuite vient le cas des deux passionsprocès. Enfin, une œuvre en tous points singulière, celle de Rivette.

Les films de De Mille et de Gastyne ont été réalisés tous les deux dans un contexte précis où conflits armés et sacrifice des guerriers devaient être exaltés. Le premier pour un combat à mener, le tournage ayant eu lieu en 1916, avant l'entrée en guerre des U. S. A., le second dans la glorification des soldats morts en sauvant la nation, au lendemain de la canonisation. Sans doute faut-il rappeler que le film américain encadre la vie de Jeanne entre deux épisodes prenant place en 1916 dans les tranchées anglaises. Un jeune officier y est la réincarnation d'un autre Anglais, Trent, contemporain de la guerre de Cent Ans et personnage totalement inventé, dont Jeanne, par ailleurs guidée par saint Michel, est une chaste amoureuse. Trent échoue à sauver Jeanne qui sublime son amour terrestre par un sacrifice patriotique. Le «Trent» de la Grande Guerre découvre dans le mur de sa casemate du côté de la Somme l'épée de Jeanne, assiste en rêve au déroulement de la vie de l'hérö̈ne et de celle de son propre double médiéval et trouve au réveil le courage d'accomplir avec enthousiasme une mission mortifère : détruire la tranchée adverse. De façon assez étrange ce film, comme l'a très bien montré Robin Blaetz ${ }^{38}$, propose une Jeanne très féminine prise entre le devoir que lui dictent saint Michel et son instinct de courageuse fille de France et de l'autre côté la tentation d'un amour partagé et sécurisant avec un tendre et mâle ennemi qui voudrait la sauver. Le personnage cependant appartient à une grande tradition johannique : celle de la Jeanne sensible et amoureuse de Schiller, tourmentée par la contradiction entre ses jeunes sens et sa mission sacrée et triomphant vertueusement pour la gloire du royaume et la sienne propre. C'est donc également la Jeanne de Verdi comme celle de Tchaïkovski, ce qui n'est

${ }^{36}$ Ida Rubinstein avait été la commanditaire de l'œuvre dont la première mondiale en allemand eut lieu à Bêle en mai 1938, et la première en français à Orléans le 6 mai 1939. Le maire d'Orléans était alors Claude Lewy ; un tract de la France enchaînée (d'extrême droite fasciste) proclama : « le clou de ces réjouissances est la représentation de la Jeanne d'Arc de Paul Claudel avec la participation de la juive Ida Rubinstein, du franc-maçon Jean Hervé, et avec la composition musicale du juif Arthur Honegger. Notre héroïne nationale est vraiment gâtée, les juifs lui font le grand honneur de l'adopter et de la séquestrer. »

${ }^{37}$ Paul Claudel n'apprécia que modérément l'interprétation d'Ingrid Bergman : « Beau tempérament, mais aucune formation» (cité par G. Antoine, Paul Claudel, ou l'enfer du génie, Paris, Laffont, 1988, p. 355)

${ }^{38}$ R. Blaetz, Strategie of containment : Joan of Arc in film..., p. 77. 
peut-être pas totalement indifférent à l'insistance de la cantatrice Geraldine Farrar à interpréter cette Pucelle, humaine et romantique, dont elle n'avait ni l'âge, ni le physique ; à l'Opéra tout passe ou presque, à l'écran c'est autre chose...

Beaucoup plus classiques, et obéissant aux normes dressées par les historiens français et non les dramaturges imaginatifs, Marco de Gastyne et son scénariste J.-J. Frappa proposèrent un résumé consensuel et orthodoxe largement inspiré, me semble-t-il, des Jeanne d'Arc d'Henri Wallon (1860) ${ }^{39}$ et de Joseph Fabre $(1883)^{40}$. La seule touche personnelle du scénario est cependant très significative. Bien que nous soyons au lendemain de la canonisation et qu'un nombreux public catholique français et étranger eût parfaitement admis une pure et simple hagiographie, les auteurs ont introduit une note patriotique et populiste fort intéressante. Jeanne, afin d'accomplir ce que ses voix lui demandaient, avait repoussé le fort brave garçon qui la demandait en mariage, Rémy Loiseau. Celui-ci, la peine au cœur, l'a laissée partir, mais fut tellement convaincu de la valeur du message guerrier qu'il devint un des premiers volontaires à arriver à Tours pour suivre sa compatriote comme porteétendard. Il meurt au siège d'Orléans et, sur l'écran, sa modeste croix tombale voit se succéder le nom des Rémy Loiseau tombés à Rocroy, Fontenoy, Valmy, Montmirail et Verdun... La mort des simples gens, paysans surtout on le sait, n'étant qu'une longue chaîne d'humbles mais fondamentaux héroïsmes qui ont permis la France d'aujourd'hui (entendons de 1928) de triompher des ennemis. De plus, à la coda du film, le bûcher de Rouen se transmue en flamme de l'Arc de triomphe... Il faut lire la fin de l'article de la Petite illustration, contemporain de la sortie du film : «Mais le sacrifice de l'hérö̈que enfant n'a pas été inutile. De lui est né la France et la flamme du bûcher de la Pucelle d'Orléans, premier soldat de France, danse et se tord encore au souffle du vent sur la tombe du Soldat inconnu, sous l'Arc de Triomphe ». Le bas de la page est occupé par la photographie de l'échafaud fumant sous-titré : Après l'holocauste ${ }^{41}$. Il s'agit à la fois du supplice de Jeanne et des millions de morts de la guerre. La chaîne tendue entre deux bornes devant le foyer évoque irrésistiblement les monuments, porteurs de longues listes, qui s'érigeaient alors dans les moindres villages. Jeanne d'Arc était devenue une sorte de Walkyrie chrétienne, hantant les champs de bataille pour consoler et prendre dans ses bras compatissants, comme après Patay, les âmes des combattants anonymes qui erraient encore nombreuses sur les champs de bataille ${ }^{42}$. La Merveilleuse vie de Jeanne d'Arc était une réponse conforme aux vœux de nombreuses familles de jeunes gens disparus à jamais : leurs enfants n'étaient pas morts épouvantés, seuls et en vain. Que le film ait

\footnotetext{
${ }^{30} \mathrm{H}$. Wallon, Jeanne d'Arc, Paris, 1860. Cet ouvrage, fort catholique mais qui intégrait les apports de la science historique, eut de très nombreuses éditions, en des formats divers, dont à partir de 1876 une série luxueuse et très bien illustrée, aujourd'hui encore recherchée.

${ }^{40} \mathrm{~J}$. Fabre, Jeanne d'Arc, libératrice de la France, Paris, 1883. J. Fabre, républicain et patriote, se battit une bonne partie de sa vie pour la célébration d'une fête républicaine en l'honneur de l'hérö̈ne nationale.

${ }^{41}$ La Petite Illustration, ${ }^{\circ}$ 408, supplément à l'Illustration du 24 novembre 1928. Les autres films traités dans cette livraison étaient : «Verdun, vision d'histoire» de Louis Poirier ; «La grande épreuve» de Duges et Ryder, deux films portant directement sur la guerre de 14-18, et... la Passion de Jeanne d'Arc de Dreyer !

${ }^{42}$ Des vitraux des années 1920-1930 montrent ce rôle intercesseur de la sainte, par exemple à Saint-Pierre-le-Moûtier ou à Clermont-Ferrand (église Sainte-Jeanne d'Arc).
} 
eu en plus une très réelle qualité plastique était une chance, non une nécessité. Son oubli vient de ce qu'il est muet.

Les marques de sentimentalité patriotique ont complètement disparu des films américains de Fleming et Preminger, mais d'autres poncifs apparaissent. Le scénario pour Fleming était tiré d'une pièce de Maxwell Anderson, Joan of Lorraine $e^{43}$, et fut surveillé de près par l'Eglise pour être conforme à une Vulgate: Jeanne commence par être canonisée et une voix-off, une sorte de Dieu le Père, intervient de temps à autre pour lier les épisodes, donner la bonne interprétation du déroulement de l'action (par exemple au moment de l'abjuration), justifier également l'inexorable marche de Jeanne vers sa douloureuse mais glorieuse fin. Robin Blaetz, qui s'intéresse au premier chef à la gender history, a relevé les contradictions entre l'indéniable présence physique, solide et adulte d'Ingrid Bergman et le traitement infantilisant qui lui est généralement réservé; elle souligne corollairement l'accent excessif mis sur la question de l'habit d'homme au cours du procès ${ }^{44}$. Mais on peut, surtout, faire une lecture assez sévère de la façon dont les autorités, l'Église et le pouvoir justifient dans ce film l'abandon de Jeanne d'Arc à son destin, en dernier argument pour son salut éternel personnel. Les scénaristes, derrière l'imagerie somptueuse, les couleurs et le son des trompettes, véhiculent une notion historique devenue discutable au $\mathrm{XX}^{\mathrm{e}}$ siècle : le rôle d'une Providence qui ôterait aux hommes la responsabilité de leurs actes, ces derniers, bons ou mauvais, faisant en quelque sorte partie d'un projet extérieur à la liberté individuelle. On rejoint presque les arguments très embarrassés de Thomas Basin, un Normand devenu évêque de Lisieux après la reconquête royale de1449-1450, qui expliquait l'apparente défaite de Jeanne d'Arc comme volonté divine devant les «démérites» et «l'ingratitude» du roi et du peuple de France ${ }^{45}$.

La Sainte Jeanne de Preminger, toute jeune et émouvante qu'elle fût, n'est pas profondément éloignée de la grosse machine hollywoodienne, le schéma n'est guère différent, s'il est plus serré et plus rigoureux. Bernard Shaw avait été adapté par Graham Greene, il ne reste vraiment rien ici de l'humour et du recul britanniques, parfois acides, du dramaturge dans le personnage moderne, candide, décidé puis traqué, campé par une Jean Seberg aussi inexpérimentée que l'hérö̈ne médiévale. Le destin de Jeanne d'Arc, telle qu'elle-même l'explique et le présente, est la suite implacable de l'enchaînement des faits. La mise en scène, les prises de vue intimistes, succédant à des tableaux bien composés, la fluidité des prises de vue ont frappé les critiques dont certains furent a posteriori dithyrambiques ${ }^{46}$, mais il est difficile de nos jours de juger sur pièce.

Il est sans doute difficile de trier «à chaud» les réflexions que suggèrent la dernière grosse machine, plus hollywoodienne que nature, celle de Besson. La masse de littérature qui a fleuri en France au moment de sa sortie est largement publicitaire et ne propose aucune réflexion de fond sur le personnage et son traitement. L'historienne s'est fort amusée de constater que le scénario, pour la première fois

${ }^{43}$ Le scénario est l'œuvre de Maxwell Anderson et Andrew Solt. Le père Paul Doncœur, jésuite, éditeur des minutes des procès, servit de conseiller scientifique et théologique.

${ }^{44} \mathrm{R}$. Blaetz, Strategie of containment, p. 83-85.

${ }^{45}$ Thomas Basin, Histoire de Charles VII, éd. Charles Samaran, Paris, Les Belles Lettres, 1933 , t. I, p. 162-163.

${ }^{46} \mathrm{Cf}$. M. Mourlet, « Jeanne d'Arc et le cinéma », supra, n 6. 
dans l'histoire du «septième art», a pris le parti délibérément anglo-bourguignon, tout en intégrant, parfois en les modifiant sans besoin réel, la plupart des poncifs sans danger de la légende johannique. L'affreuse Yolande d'Aragon, sans foi ni loi, tire les grosses ficelles ; Charles VII (J. Malkovitch n'y est pour rien) se révèle encore plus veule que chez Ucicky. Le petit soldat Jeanne est acculé à reconnaître qu'elle s'est trompée sur toute la ligne, abusée par sa foi et ses rancœurs d'origine traumatiques, donc le tribunal de Rouen avait totalement raison, Dieu n'était pas de son côté. Que l'héroïne soit une silhouette attachante, les batailles fracassantes, les compagnons conformes à la Vulgate (soudards mais respectueux), les décors plutôt réussis, il manquera toujours à ce scénario une épaisseur simplement humaine à la Pucelle et plus encore, la moindre étincelle de son intelligence. Sa mort reste une énigme.

À une génération ou presque d'écart, Dreyer et Bresson ont pris les partis classiques de l'unité de lieu, d'action et presque de temps : la prison, le procès, les dernières semaines. Avec le recul, la parenté apparaît de plus en plus certaine entre les deux films ${ }^{47}$. Leur originalité réciproque s'avère également éclatante, d'autant qu'il n'y a aucun plagiat de Bresson à Dreyer, mais une source historique commune, les textes du procès de condamnation. La vision première de Dreyer avait été provoquée par Joseph Deltheil ; il ne transparaît rien ou presque de la rondeur rustique du personnage primitif de Jeanne dans la rigueur souffrante, par ailleurs assez démonstrative, malgré la sincère recherche d'intériorité, du rôle donné à Falconetti et relu par Pierre Champion dans les minutes des procès. Harcelée par un tribunal composé de très fortes personnalités, à la fin Jeanne est broyée, le spectateur est terrifié, la rédemption de tous est douteuse. La survie semble ne devoir s'imprimer que dans la mémoire des spectateurs de la Passion de Jeanne d'Arc. Quant à Bresson, il a surtout cherché à montrer la cohérence de l'attitude de Jeanne pendant l'entière durée de son aventure terrestre. Son hérö̈ne, au long des scènes du Procès qui viennent en répétition, prend de plus en plus de consistance sans que les effets soient le moins du monde appuyés. L'apparente insignifiance du physique de Florence Carrez(-Delay) dissimule une force croissante du personnage ${ }^{48}$. Une évidence se fait jour, peu à peu: Jeanne dispose d'une vie intérieure mystérieuse qui échappe davantage à chaque séance à la curiosité et à la compréhension trop terrestre de ses juges. Ceux-ci échouent dans toutes leurs tentatives pour réduire l'hérö̈ne aux catégories de la raison, sa mort est leur faillite - et sa victoire. On peut penser que Bresson a ici frôlé l'indicible.

Sans doute, n'est-ce pas diminuer le mérite des deux cinéastes que de souligner l'efficacité actuelle des scènes de procès. Les mots et les silences ont une valeur d'évocation supérieure à la représentation réaliste. Le théâtre clos du prétoire referme le regard sur les protagonistes et peut cerner une vérité abstraite par des

${ }^{47}$ J. Sémolué, «Passion et Procès (de Dreyer à Bresson) », Études cinématographiques, $\mathrm{n}^{\circ} 18$ 19, 1962, p. 98-107: «[L’] établissement de la construction sur la vie intérieure explique leurs conceptions de l'interprétation. Leurs déclarations sont presque interchangeables » (p. 99).

${ }^{48} \mathrm{C}$ 'est le processus rigoureusement inverse chez Luc Besson : construite par ses visions, sa ténacité et ses succès, la personnalité publique de Jeanne se dissout dans le doute et finit par l'insignifiance; sa mort n'est qu'un spectacle coloré. 
phrases, mais, simultanément, il laisse sortir du cadre du langage l'imagination du spectateur nourrie depuis l'enfance des images traditionnelles de la «bergère », de la fille en armes, des batailles et des étapes de l'épopée johannique. Nul besoin de représenter le sacre : Ingres ou Jean-Paul Laurens s'imposent à nous. Jeanne devant les Tourelles, ce sont les fresques de Lenepveu au Panthéon, popularisées par les livres de classe puis les bandes dessinées. Nous avons rencontré tant de fois la Pucelle à cheval dans les rues de Paris, Reims, Orléans, Chinon, et l'avons contemplée en prière dans des centaines, voire des milliers d'humbles églises de France.... Une phrase et l'écran se peuple d'images, personnelles à chacun.

Jacques Rivette a tenté de concilier les deux voies de la représentation: l'évocation par des témoignages et des récits, en parallèle à la peinture des faits de l'histoire en leur temps. La gageure a abouti à une œuvre hybride, déconcertante et difficile, d'où un échec public certain pour le réalisateur, malgré une réussite personnelle et méritée de l'actrice, Sandrine Bonnaire. Aucune véritable concession n'est faite au spectacle, pas plus des Batailles que des Prisons; pas d'Hollywood à Chinon, aucune facilité d'un procès de Rouen au dénouement inéluctable. Le puzzle des enfances et de la vie publique de Jeanne la Pucelle, reconstituées par les interventions extérieures des témoins de 1456, est plus complexe que celui de Bresson, mais il s'encastre presque trop bien. Le rythme est au fil des saisons : il fait glacial, en cet hiver de 1428-1429, pour traverser la Lorraine, tiède et fleuri en mai dans les jardins d'Orléans, chaud à Reims en juillet mais frais dans la cathédrale, humide sur les bords de Loire en un long hiver d'attente, oppressant dans la tour de Rouen. Les ennemis sont partout, Jeanne, en lutte contre tout et tous depuis Domrémy jusqu'à Rouen, est à l'antithèse du personnage de Fleming : elle n'est jamais vraiment héroïque, mais en justification permanente de son droit à participer à sa propre épopée. Les respirations, le séjour près des dames de Luxembourg, ne sont pas porteuses d'espoir. La conception de Rivette est foncièrement pessimiste, anxiogène plus encore que celle de Dreyer : Jeanne a-t-elle vécu et est-elle morte pour quelque chose ? L'intériorité de Jeanne, sa solitude l'accompagnent et l'isolent, comme son armure, en toutes circonstances, empêchant une quelconque assimilation des spectateurs, aussi bien à sa gloire qu'à son calvaire. Cette retenue constante, qui n'est pas assumée sans montrer cependant de fugitives pointes d'admiration, ne se retrouve dans aucun autre des films sur Jeanne d'Arc, au cours desquels il y a toujours un moment où l'on peut vibrer ou souffrir en communion, voire rire en complicité avec un personnage suffisamment proche pour être presque contemporain. La foi de la Jeanne de Rivette est l'objet d'une tentative de dissection, alors qu'on sait qu'on n'a pas encore trouvé l'âme au bout du scalpel. Le film de Rivette est d'un temps où l'on a peur de la foi et de ses excès, des intégrismes et des fous de Dieu, un temps où l'on voudrait pouvoir cerner le virus du mysticisme, considéré comme une pathologie, plutôt que reconnaître les limites médiévales de la connaissance rationnelle. La conception de Jeanne m'y paraît aussi étroitement « datée » que celles de Cecil B. De Mille ou de Fleming.

Le cinéma, quand il n'est pas œuvre de tâcheron, est création d'artiste et, dans tous les cas, il est reflet du temps contemporain, c'est sa richesse. Il s'attaque avec le thème de Jeanne d'Arc à la complexité d'un personnage à la fois humain et 
mythique, totalement hors des normes anciennes ou actuelles. Il me semble, opinion subjective d'une historienne médiéviste, qu'il ne peut sortir vainqueur de la confrontation, dévoré par la complexité de son sujet même.

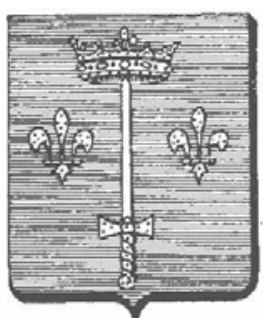

